\title{
Overwhelming sepsis presenting as sudden unexpected death
}

\author{
N Sharief, K Khan, P Conlan
}

\begin{abstract}
Four children, including three infants, who died suddenly and unexpectedly are described. In three of the cases group A $\beta$ haemolytic streptococcus was cultured and in the fourth Streptococcus pneumoniae. The organism was grown from multiple sites including blood in two of them. Without microbiological investigation the diagnosis would have been sudden unexpected death syndrome. (Arch Dis Child 1993; 69: 381-383)
\end{abstract}

Sudden infant death is a well known clinical entity and seems to be multifactorial in origin. ${ }^{1}$ Overwhelming sepsis as a cause of sudden unexpected death in children is well recognised and alleged to be a cause in up to $7 \%$ of cases. ${ }^{2}$ The literature includes very few reports describing the association between overwhelming sepsis and sudden unexpected death, although group B streptococcus has been previously implicated. ${ }^{3}$ We hereby describe four cases of sudden unexpected death in infancy and childhood that were due to overwhelming sepsis and presented over a period of eight weeks.

\section{Case reports}

CASE 1

A previously well baby was found to be snuffly at the routine check at 6 weeks of age. Two weeks later, over a 24 period she was reluctant to feed and early that morning she woke up screaming and crying. Suddenly she became limp and cyanotic. She was resuscitated by her parents and taken to hospital where she was found to be dead on arrival.

Postmortem examination showed the trachea to contain some frothy fluid. The lungs were congested with subpleural petechial haemorrhages in the interlobular fissures; no other abnormalities were found. Microbiology confirmed group A $\beta$ haemolytic streptococcus (GABHS) from the blood, lungs, and heart. The pathologist felt that the cause of death was acute bronchiolitis.

Basildon Hospital, Nether Mayne, Basildon, Essex SS16 5NL

N Sharief

K Khan

P Conlan

Correspondence to:

Dr Khan.

Accepted 27 April 1993
CASE 2

A child aged 3.5 months, known to be on the 'at risk' register (because of concern about maternal skills), was put to sleep in his pram early one evening. A few hours later he was found dead in the prone position. No preceding symptoms were reported.

Postmortem examination revealed congested lungs with surface petechiae, as well as congested liver, spleen, and thymus. Streptococcus pneumoniae was isolated from the blood, lungs, liver, brain, heart, and trachea.

\section{CASE 3}

A child of 8.5 months who had been previously well (apart from conjunctivitis at the age of 6 weeks) was seen by his general practitioner a week before his death for an upper respiratory tract infection. He appeared well when being put to bed one evening, he was found cold and lifeless in the morning.

On postmortem examination the lungs were congested with surface petechiae. The brain, liver, and thymus were also congested. GABHS was isolated from the brain, lungs, trachea, spleen, heart, and stool.

\section{CASE 4}

A 4 year old child was seen by her general practitioner because of a sore throat. For the next two weeks she was noticed to be chesty and listless. On the night of her death she was put to bed but kept getting up and falling out of bed. She was found lifeless in the morning.

Postmortem examination showed mild meningeal congestion and a tiny soft polypoid nodule $0.6 \mathrm{~cm}$ in diameter attached to the meninges above the tentorium cerebeli. The trachea and bronchi were congested and filled with pink frothy fluid. Both lungs were deep purple in colour with scattered subpleural petechial haemorrhages. The heart also showed some pericardial petechiae on the anterior wall of the left ventricle. GABHS was cultured from the trachea, lungs, and spleen. Histology of the lungs was thought to suggest bacterial infection superimposed on viral pneumonitis.

\section{Discussion}

In all our cases the death of the child was sudden and unexpected. In three of the cases GABHS was cultured from multiple sites, including blood in one of them. In the fourth $S$ pneumoniae was grown from multiple sites including blood. Although the presence of bacterial isolates at postmortem examination have been questioned, ${ }^{4}$ we feel confident that the isolation of the same organism from multiple sites is good evidence of the cause of death in these children. Histological evidence of a pulmonary cause of death was noted in two of our cases but was unremarkable in the other two. It is worth noting that these four children presented within a period of eight weeks in a fairly small geographical area, covering a population of 281800 . Clustering 
of GABHS has been reported ${ }^{5}$ and clustering of sudden unexpected death is also well known, indeed the geographical pattern and seasonal variation of sudden unexpected death is itself suggestive of an infective process. ${ }^{6}$ In all our cases postmortem examination did not show a convincing cause of death and without microbiological evidence the diagnosis would have been sudden unexpected death.

1 Marshall TA. Sudden infant death syndrome - update. $f S C$ Med Assoc 1985; 81: 605-8.

2 Norman MG, Taylor GP, Clarke LA. Sudden unexpected natural death in children. Pediatr Pathol 1990; 10: 769-84

3 Barnham M, Henderson DC. Group B streptococcal infection presenting as sudden death in infancy. Arch Dis Child 1987; 62: 419-20.

4 Gilbert R, Rudd P, Berry J, et al. Combined effect of infection and heavy wrapping on the risk of sudden unexpected and heavy wrapping on the risk of sudden

5 Schwartz B, Facklam RR, Breiman RF. Changing epidemiology of group A streptococcal infection in the United States of America. Lancet 1990; 336: 1167-71.

6 Knox EG, Lancashire RJ. Cot death in Birmingham. $f$ Public Health Med 1990; 13: 142-50.

\section{Commentary}

In the 19th century Louis Pasteur identified cocci in chains in the uterus and blood of women dying from puerperal sepsis. By the 1930s, studies of illness related to Streptococcus pyogenes revealed a highly virulent organism capable of causing severe, often fatal invasive disease. ${ }^{12}$ It is therefore remarkable that in a trend that started before the introduction of effective antibiotics, the mortality associated with group A $\beta$ haemolytic streptococcus (GABHS) has continued to decrease over the last 50 years, and bacteraemia with the organism has become a rare clinical entity. ${ }^{34}$ Today, although GABHS is among the most common pathogenic bacteria isolated from children, most paediatricians associate the organism with an acute pharyngitis or tonsillitis, rarely seeing acute suppurative disease, glomerulonephritis, or acute rheumatic fever. In this paper Sharief and colleagues report a cluster of three patients who died from overwhelming GABHS infection. Interestingly, all the cases occurred within eight weeks of each other in a small geographical area. Their paper is important in highlighting a recent change in not only the incidence of GABHS bacteraemia but also in the clinical spectrum of the disease. It now appears that the previous trend has reversed, with increasing reports of rheumatic fever and invasive infection among children in Europe and the United States. ${ }^{3-5}$

These recent outbreaks have a number of similarities with those described over 50 years ago, with the majority of the patients with GABHS bacteraemia presenting with fever and sore throat, cellulitis, osteomyelitis, septic arthritis, pneumonia, meningitis, and overwhelming sepsis. ${ }^{36}$ In contrast, however, the mortality associated with the resurgent disease has been significantly lower (7-12\%) than the $76 \%$ observed in the preantibiotic era. ${ }^{136}$ Although the disease has occurred after varicella and in association with congenital immunodeficiency or malignancy, the majority of cases have not had an underlying abnormality.
Among these new reports of severe GABHS infection there have been a number of cases of a toxic shock-like syndrome associated with the production of streptococcal toxins. ${ }^{45}$ These pyrogenic exotoxins enhance host susceptibility to endotoxin ${ }^{7}$ and like staphylococcal toxic shock syndrome toxins ${ }^{8}$ act as superantigens in their ability to expand specific $T$ cell families and provoke cytokine release. $^{5}$ Interestingly, it seems that this apparently new clinical entity of toxic shocklike syndrome was described over 60 years ago. In a report of an epidemic of severe scarlet fever in Yunnafu, China (1921-2), Weech identified both severe toxic and invasive septicaemic forms of the disease that were associated with throat cultures positive for haemolytic streptococci. ${ }^{2}$ It is astonishing that in this epidemic, the entire population of 200000 was affected and 50000 deaths occurred. Therefore, if the clinical manifestations of this resurgent streptococcal disease are not new, has an old, virulent strain of GABHS re-emerged, or has a new variant developed?

To answer this question it important to appreciate that the GABHS has a number of virulence factors. The role of pyrogenic exotoxins remains hotly debated, ${ }^{9} 10$ but there are many other factors including secreted proteins such as streptokinase, hyaluronidase and the haemolysins, in addition to surface proteins such as $\mathrm{C5a}$ peptidase, $\mathbf{M}$ proteins, and the immunoglobulin Fc receptor protein. Of these, the $M$ proteins are thought to be particularly essential for the survival of GABHS within the host. These are fibrillar molecules extending from the bacterial cell surface, ${ }^{11}$ which facilitate adherence to epithelial cells and inhibit effective complement mediated neutrophil phagocytosis. ${ }^{7} 1112$ In a study of isolates collected between 1972 and 1988 by the Centers for Disease Control in Atlanta, Schwartz and coworkers demonstrated a significant increase in the proportion of $M$ types 1, 3, and $18 .{ }^{4}$ In England, $M$ type 1 strains have increased from $1 \%$ in 1980 to $30 \%$ of all GABHS isolates in $1987,,^{13}$ and have largely been responsible for the dramatic increase in invasive disease in Sweden and Norway. ${ }^{14}$ Therefore GABHS strains expressing specific $M$ proteins seem to account for the resurgence of invasive disease in both Europe and the United States.

Using multilocus enzyme electrophoresis in conjunction with $\mathbf{M}$ protein serotyping, Musser and coworkers have confirmed that nearly half of invasive disease episodes in Europe and the USA are caused by $M$ type 1 and $M$ type 3 strains, and have demonstrated that these strains are derived from closely related genetic clones, denoted ET 1 and 2 respectively. ${ }^{15}$ After analysis of isolates from the 1920s and 1930s, Musser et al have gone on to suggest that these clones have emerged through changes in the older strains that occurred sometime in the 1960 s and 1970 s, possibly driven by the low level of herd immunity. ${ }^{10}$ Restriction fragment profile studies support this hypothesis, ${ }^{9}$ and add to the weight of evidence suggesting that, after their 